Document downloaded from:

http://hdl.handle.net/10251/30405

This paper must be cited as:

Ginestar Peiro, D.; Thome Coppo, NJ.; Coll Aliaga, PDC.; Sánchez Juan, E. (2012). Drazin inverse based numerical methods for singular linear differential systems. ADVANCES IN ENGINEERING SOFTWARE. (50):37-43. doi:10.1016/j.advengsoft.2012.04.001.

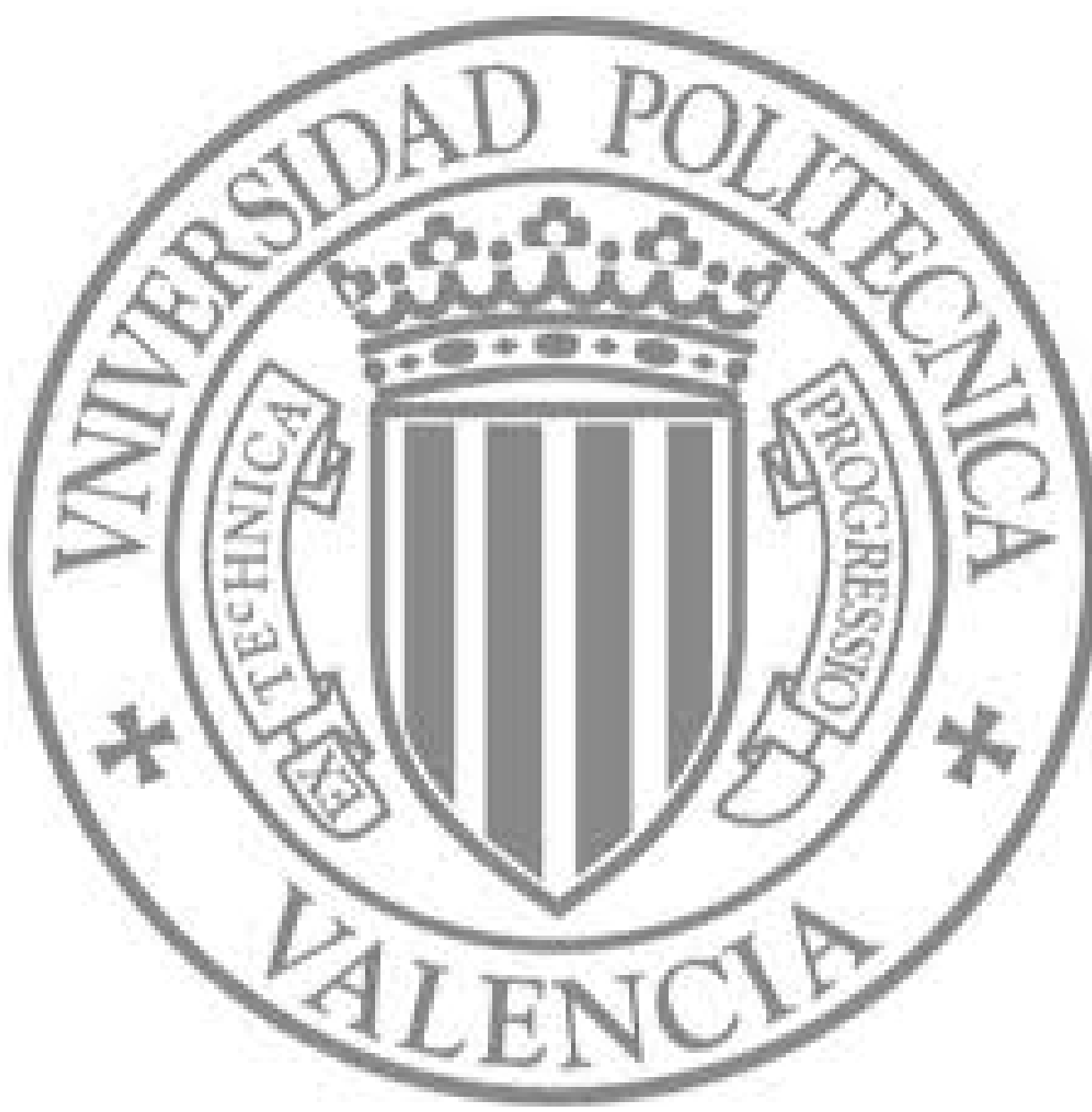

The final publication is available at

http://dx.doi.org/10.1016/j.advengsoft.2012.04.001

Copyright ELSEVIER SCI LTD 


\title{
Drazin inverse based numerical methods for singular linear differential systems
}

\author{
C. Coll, D. Ginestar, E. Sánchez and N. Thome \\ Instituto de Matemática Multidisciplinar \\ Universitat Politècnica de València, Spain
}

\begin{abstract}
In this paper, numerical methods for the solution of linear singular differential system are analysed. The numerical solution of initial value problems by means of a finite difference approach and a possible implementation of the Drazin inverse vector product is discussed. Examples of index- 1 and index- 2 differential algebraic equations have been studied numerically.
\end{abstract}

Keywords: Singular systems, numerical methods, shuffle algorithm, Drazin inverse.

\section{Introduction}

Singular differential systems are systems of ordinary differential equations (ODEs) that cannot be expressed in their normal form. Special cases of such systems defined by differential algebraic equations (DAEs) arise in the mathematical modelling of problems from engineering and science such as electrical circuit design, optimal control, incompressible fluids, molecular dy- 
namics, large-scale interconnected systems, etc [3]. Singular linear systems are a particular class of these systems, which consist of a system of differential equations where the derivatives are pre-multiplied by a singular matrix. For example, the spatial discretization of the Stokes equation describing the flow of an incompressible fluid by finite differences on a uniform staggered grid leads to a singular system. The vibration of a damped mass-spring system with holonomic constraints is described by a singular system, etc. [2]. The solution of singular systems has been studied by several authors. For instance, in [5] an analytical solution for continuous systems using the Drazin inverse was presented. In [19] a solution in the invariant-time case is given using the Weierstrass form [7] and in [16] the solution of singular systems was treated from a numerical point of view. Some surveys of this kind of systems can be found in $[3,10]$. In the analytical and numerical treatments of a DAE, the index plays an important role [3] and it provides useful information about the mathematical structure and potential complications in the solution of the DAE. The number of repetitions needed for the transformation of the DAE into its related ODE is called the differential index of the DAE. In general, initial value problems associated to a linear constant coefficient singular system have no solution or the solution can not be unique. Some conditions have to be fulfilled in order to get a unique solution, namely regularity conditions.

The usual technique to solve singular systems satisfying the regularity condition requires the computation of two Drazin inverses [5]. A numerical method to provide information about the solvability of the singular system is provided by Luenberger' shuffle algorithm [12]. This method is based on a process of triangularization of the system matrices which destroys the possible sparsity of the matrices. 
In this paper, we investigate the use of numerical techniques to solve singular systems based on the Drazin inverse formulation of the system solution. Some computational aspects of the Drazin inverse are indicated in [17]. However, the main operation used in the numerical methods proposed is the Drazin inverse vector product. This product can be efficiently computed by several algorithms based on the matrix vector product and the Drazin inverse matrix is not explicitely constructed.

The outline of the paper is as follows. Section 2 presents some preliminaries of singular systems and introduces the concept of admisible initial conditions for a solvable singular system. In section 3 numerical methods for the solution of an initial value problem associated with a singular system are presented. A possible implementation of the Drazin inverse vector product is also given. In section 4, numerical results are discussed for different linear singular systems. Finally, the main conclusions of the paper are given in section 5 .

\section{Singular systems}

A linear singular system is given by

$$
E x^{\prime}(t)=A x(t)+f(t)
$$

with $E, A \in \mathbb{R}^{n \times n}$, being $E$ a singular matrix. If the singular system satisfies the regularity condition, that is, $\operatorname{det}(\lambda E-A) \neq 0$ for at least one scalar $\lambda$, then a unique solution of an initial value problem may be obtained by specifying a suitable set of initial conditions.

If the matrix $A$ is nonsingular, the regularity condition holds. By means of linear algebra techniques, an explicit solution of a singular system can be 
obtained [5]. This solution involves Drazin inverses of the state matrices. Recall that the Drazin inverse of a matrix $A \in \mathbb{R}^{n \times n}$ is the matrix $A^{D}$ satisfying:

(i) $A^{D} A A^{D}=A^{D}$,

(ii) $A A^{D}=A^{D} A$ and

(iii) $A^{k+1} A^{D}=A^{k}$, where $k=\operatorname{ind}(A)$ is the index of $A$, which is the smallest nonnegative integer such that $\operatorname{rank}\left(A^{k}\right)=\operatorname{rank}\left(A^{k+1}\right)$.

In this paper, we analyse the solution of the following initial value problem

$$
E x^{\prime}(t)=A x(t)+f(t), x\left(t_{0}\right)=x_{0} .
$$

Without loss of generality, we can consider $t_{0}=0$.

The concept of index is useful to characterize DAEs. Intuitively, the index is used for measuring the distance from a DAE to its related ordinary differential equation. If the singular system is solvable, the index of the matrix affecting the derivative is closely related to index of the system [3]. Naturally, the index of the DAE (1) is the same as the index of the matrix $E[8]$.

\subsection{The homogeneous problem}

Consider the following singular initial value problem

$$
E x^{\prime}(t)=A x(t), \quad x(0)=x_{0} .
$$

The solution of this singular system is given by [5]

$$
x(t)=e^{\hat{E}^{D} \hat{A} t} \hat{E}^{D} \hat{E} x_{0},
$$


where $\hat{E}=(\lambda E-A)^{-1} E, \hat{A}=(\lambda E-A)^{-1} A$ and the initial vector $x_{0}$ is admissible, that is, it fulfills the condition

$$
\left(I-\hat{E}^{D} \hat{E}\right) x_{0}=0
$$

The solution (3) is independent of the scalar $\lambda$ used to construct $\hat{E}$ and $\hat{A}$. To show this independence, we denote $\hat{E}_{\alpha}=(\alpha E-A)^{-1} E$, for $\alpha \in \mathbb{R}$, and it is sufficient to prove that

$\hat{E}_{\alpha}^{D} \hat{E}_{\alpha}=\hat{E}_{\beta}^{D} \hat{E}_{\beta}$ and $\hat{E}_{\alpha}^{D} \hat{A}_{\alpha}=\hat{E}_{\beta}^{D} \hat{A}_{\beta}$ for all scalar $\alpha, \beta$. In fact

$$
\begin{aligned}
& \left.\hat{E}_{\alpha}^{D} \hat{E}_{\alpha}=\left((\beta E-A)^{-1}(\alpha E-A)\right)^{-1}(\beta E-A)^{-1} E\right)^{D} \hat{E}_{\alpha}= \\
& \left(\left(\alpha \hat{E}_{\beta}-\hat{A}_{\beta}\right)^{-1} \hat{E}_{\beta}\right)^{D} \hat{E}_{\alpha}=\hat{E}_{\beta}^{D}(\beta E-A)^{-1}(\alpha E-A)(\alpha E-A)^{-1} E=\hat{E}_{\beta}^{D} \hat{E}_{\beta} .
\end{aligned}
$$

The proof of $\hat{E}_{\alpha}^{D} \hat{A}_{\alpha}=\hat{E}_{\beta}^{D} \hat{A}_{\beta}$ is similar.

If the matrix $A$ is nonsingular, there exists at least $\lambda=0$, such that $\operatorname{det}(\lambda E-A) \neq 0$. In this case, without loss of generality, we can assume that the solution of initial value problem (2) can be obtained using

$$
x(t)=e^{\bar{E}^{D} t} \bar{E}^{D} \bar{E} x_{0} .
$$

with $\bar{E}=A^{-1} E$. Since the solution is uniquely determined, we can use the initial value problem of ODEs associated to the original problem (2) obtained by differentiation of (5). This problem is given by

$$
x^{\prime}(t)=\bar{E}^{D} x(t), x(0)=x_{0} .
$$




\subsection{The non-homogeneous problem}

We now study the non-homogeneous case, that is, let us consider the singular system

$$
E x^{\prime}(t)=A x(t)+f(t)
$$

with the initial condition $x(0)=x_{0}$. When $A$ is nonsingular, the solution of this system is given by means of the same matrix $\bar{E}=A^{-1} E$ as follows

$$
\begin{aligned}
x(t) & =e^{\bar{E}^{D} t} \bar{E}^{D} \bar{E} x_{0}+e^{\bar{E}^{D} t} \bar{E}^{D} \int_{0}^{t} e^{-\bar{E}^{D} s} \bar{f}(s) d s \\
& -\left(I-\bar{E}^{D} \bar{E}\right) \sum_{j=0}^{k-1} \bar{E}^{j} \frac{d^{j}}{d t^{j}} \bar{f}(t),
\end{aligned}
$$

where $\bar{f}(t)=A^{-1} f(t)$ and $k=\operatorname{ind}(\bar{E})$. Moreover, in order to satisfy $x(0)=$ $x_{0}$, the initial condition must satisfy the following relation

$$
\left(I-\bar{E}^{D} \bar{E}\right) x_{0}=-\left(I-\bar{E}^{D} \bar{E}\right) \sum_{j=0}^{k-1} \bar{E}^{j} \frac{d^{j}}{d t^{j}} \bar{f}(0),
$$

which is the admissibility condition for the non-homogeneous problem.

Note that, using $\bar{E}^{D}\left(I-\bar{E}^{D} \bar{E}\right)=O$, we have that the solution (8) satisfies

$$
\bar{E}^{D} x(t)=\bar{E}^{D} e^{\bar{E}^{D} t} \bar{E}^{D} \bar{E} x_{0}+\bar{E}^{D} e^{\bar{E}^{D} t} \bar{E}^{D} \int_{0}^{t} e^{\bar{E}^{D} s} \bar{f}(s) d s .
$$

Hence, (8) is also the solution of the following system of ODEs associated to the singular system $(7)$

$$
x^{\prime}(t)=\bar{E}^{D} x(t)+\bar{E}^{D} \bar{f}(t)-\left(I-\bar{E}^{D} \bar{E}\right) \sum_{j=0}^{k-1} \bar{E}^{j} \frac{d^{j+1}}{d t^{j+1}} \bar{f}(t) .
$$




\section{Numerical methods for a singular system}

In this section, we propose numerical methods for an initial value problem associated with a singular system based on the Dazin inverse formulation of its solution. Two different strategies are proposed. In (5) and (8), the explicit solutions for a solvable singular system were given. Using these expressions, we propose a method to approximate these solutions by using standard explicit numerical techniques. An alternate way to solve the initial value problem (1), is to use the associated ordinary differential problem (10) and to apply a numerical algorithm by discretizing the derivative.

Let us consider the analytic solution (5) of the homogeneous singular system (2) with an admissible initial condition $x_{0}$. If the time is discretized as $t_{n}=t_{0}+n \Delta t$ and we consider the first order approximation of the exponential matrix

$$
e^{\bar{E}^{D} \Delta t}=I+\bar{E}^{D} \Delta t+O\left(\Delta t^{2}\right)
$$

we obtain the first order explicit method

$$
x_{n}=\left(I+\Delta t \bar{E}^{D}\right) \bar{E}^{D} \bar{E} x_{n-1}, \quad n \geq 1
$$

In order to assure the consistency of the solution during the iteration process we have to prove that $x_{n}$ satisfies the admissible initial condition (4) for all $n \geq 1$. In fact, using properties of the Drazin inverse we have that

$$
\left(I-\bar{E}^{D} \bar{E}\right) x_{n}=\left(I-\bar{E}^{D} \bar{E}\right)\left(I+\Delta t \bar{E}^{D}\right) \bar{E}^{D} \bar{E} x_{n-1}=0 .
$$

One difference between the numerical methods for an ODE and the corresponding numerical method for a singular system is that a consistent initial 
value has to be computed to start the integration in each step.

If we now consider the system of ODEs (6) and we use the standard forward Euler method, we obtain the numerical scheme

$$
x_{n}=\left(I+\Delta t \bar{E}^{D}\right) x_{n-1}, n \geq 1
$$

which is also a consistent numerical scheme.

For the non-homogeneous case, we consider the analytical solution (8) and we use the approximation (11) for the matrix exponential. We also consider a rectangular approximation for the integral and the approximation for the derivative

$$
\frac{d^{j} \bar{f}}{d t^{j}}\left(t_{n}\right)=\bar{f}_{n}^{j} \approx \frac{\bar{f}_{n+1}^{j-1}-\bar{f}_{n}^{j-1}}{\Delta t}
$$

being $\bar{f}\left(t_{n}\right)=\bar{f}_{n}^{0}$. We obtain the numerical scheme

$x_{n}=\left(I+\Delta t \bar{E}^{D}\right) \bar{E} \bar{E}^{D} x_{n-1}+\left(I+\Delta t \bar{E}^{D}\right) \bar{E}^{D} \Delta t \bar{f}_{n-1}-\left(I-\bar{E}^{D} \bar{E}\right) \sum_{j=0}^{k-1} \bar{E}^{j} \bar{f}_{n}^{j}$.

If we use the system of ODEs (10) and apply the forward Euler method, we obtain the numerical scheme

$$
x_{n}=\left(I+\Delta t \bar{E}^{D}\right) x_{n-1}+\Delta t \bar{E}^{D} \bar{f}_{n-1}-\left(I-\bar{E}^{D} \bar{E}\right) \sum_{j=0}^{k-1} \bar{E}^{j}\left(\bar{f}_{n}^{j}-\bar{f}_{n-1}^{j}\right) .
$$

Both numerical schemes (14) and (15) satisfy the approximate consistency condition

$$
\left(I-\bar{E}^{D} \bar{E}\right) x_{n}=-\left(I-\bar{E}^{D} \bar{E}\right) \sum_{j=0}^{k-1} \bar{E}^{j} \bar{f}_{n}^{j}
$$




\subsection{Computation of the Drazin inverse vector product}

The numerical schemes proposed above can be easily implemented once we have a suitable procedure to compute the Drazin inverse vector product. Several methods can be applied to compute this operation, such as the semi-iterative methods [6, 14], a Bi-CG type method [15] and the DGMRES method [13]. We have used a Newton method proposed in [11] and [18] that computes an approximation for the Drazin inverse of a matrix $A$ with index $k$, using the recurrence

$$
\begin{aligned}
& A_{0}=\alpha A^{l}, \alpha>0, l \geq k, \\
& A_{n}=2 A_{n-1}-A_{n-1} A A_{n-1}, n \geq 1 .
\end{aligned}
$$

To implement the product $A^{D} b$, the following recurrence is used

$$
\begin{aligned}
& y_{0}=\alpha A^{l} b, c_{0}=A y_{0}, z_{0}=A_{0} c_{0} \\
& y_{n+1}=2 y_{n}-z_{n}, c_{n+1}=A y_{n+1}, z_{n+1}=A_{n+1} c_{n+1}, n \geq 0 .
\end{aligned}
$$

The convergence of the method is quadratic [11] and to use the method we have selected the value $\alpha=0.01$.

\section{Stability problem}

In this section we study the stability of singular system (2) and the proposed numerical methods. We recall that a singular system $E x^{\prime}(t)=A x(t)$ is asymptotically stable iff all $\lambda^{\prime}$ s such that $\operatorname{det}(\lambda E-A)=0$ have negative real part.

Note that, the system $(2)$ is asymptotically stable when $\operatorname{det}(A) \neq 0$. In 
this case we use $\bar{E}=A^{-1} E$ and the new system is given by

$$
\bar{E} x^{\prime}(t)=x(t)
$$

System (16) is asymptotically stable iff all solutions of $\operatorname{det}(\lambda \bar{E}-I)=0$

have negative real part, that is, if we take $\lambda=\frac{1}{\alpha}, \alpha \neq 0$ the equality $\operatorname{det}(\alpha I-\bar{E})=0$ gives the nonzero eigenvalues of matrix $\bar{E}$ and they have to have negative real part.

In general, an arbitrary system is said to be bounded input-bounded output (BIBO) stable if and only if every bounded input produces the bounded output.

\subsection{Stability of numerical methods}

An asymptotic stability condition for numerical methods proposed for system (2) is obtained if we consider the numerical scheme as a discrete system. The discrete system

$$
x_{n}=A x_{n-1}
$$

is asymptotically stable iff the eigenvalues of matrix $A$ are inside the unit circle. In the same way as for the continuous case, a discrete system is said to be BIBO stable if and only if every bounded input produces the bounded output.

We now analyse the numerical schemes proposed for problem (10).

Case I: Using the first order approximation of the exponential matrix. For this case the numerical scheme is given by

$$
x_{n}=\left(I+\Delta t \bar{E}^{D}\right) \bar{E}^{D} \bar{E} x_{n-1}, \quad n \geq 1 .
$$


The above discrete system is asymptotically stable iff all $\lambda$ such that

$$
\operatorname{det}\left(\lambda I-\left(I+\Delta t \bar{E}^{D}\right) \bar{E}^{D} \bar{E}\right)=\operatorname{det}\left(\lambda I-\Delta t \bar{E}^{D}-\bar{E}^{D} \bar{E}\right)=0
$$

satisfy $|\lambda|<1$. If $|\lambda|=1$ then the system is stable. Note that, we have to study the eigenvalues of the matrix $\Delta t \bar{E}^{D}+\bar{E}^{D} \bar{E}$.

Case II: Using the standard forward Euler method. For the ordinary differential equation (6) we obtain the numerical scheme is given by

$$
x_{n}=\left(I+\Delta t \bar{E}^{D}\right) x_{n-1}, n \geq 1 .
$$

The above discrete system is asymptotically stable iff all $\lambda$ such that

$$
\operatorname{det}\left(\lambda I-\Delta t \bar{E}^{D}-I\right)=0
$$

satisfy $|\lambda|<1$. If $|\lambda|=1$ then the system is stable. Note that in this case we have to study the eigenvalues of the matrix $\Delta t \bar{E}^{D}+I$.

Note that, the core-nilpotent decomposition of the matrix $\bar{E}$ is given by

$$
\bar{E}=S\left(\begin{array}{cc}
C & O \\
O & N
\end{array}\right) S^{-1}
$$

where the matrices $S$ and $C$ are nonsingular and $N$ is a nilpotent matrix with $\operatorname{ind}(N)=\operatorname{ind}(E)[4]$. Then,

$$
\bar{E}^{D}=S\left(\begin{array}{cc}
C^{-1} & O \\
O & O
\end{array}\right) S^{-1}, \quad \Delta t \bar{E}^{D}+\bar{E}^{D} \bar{E}=S\left(\begin{array}{cc}
\Delta t C^{-1}+I & O \\
O & O
\end{array}\right) S^{-1}
$$


and

$$
\Delta t \bar{E}^{D}+I=S\left(\begin{array}{cc}
\Delta t C^{-1}+I & O \\
O & I
\end{array}\right) S^{-1}
$$

It is now clear that the spectrum of the above matrices are related.

\section{$5 \quad$ Numerical Results}

\subsection{First example}

To test the performance of the numerical methods proposed, we will study the singular system

$$
\left(\begin{array}{cc}
L_{1} & \sqrt{L_{1} L_{2}} \\
\sqrt{L_{1} L_{2}} & L_{2}
\end{array}\right)\left(\begin{array}{c}
x_{1}^{\prime} \\
x_{2}^{\prime}
\end{array}\right)=-\left(\begin{array}{cc}
R_{1} & 0 \\
0 & R_{2}
\end{array}\right)\left(\begin{array}{c}
x_{1} \\
x_{2}
\end{array}\right)+\left(\begin{array}{c}
V \sin (w t) \\
0
\end{array}\right),
$$

that models the electric circuit associated with an ideal transformer shown

in Figure 1. Where $x=\left(x_{1}, x_{2}\right)^{T}$ is the currents vector $\left(I_{1}, I_{2}\right)^{T}$. This is a singular system of index $k=1$.

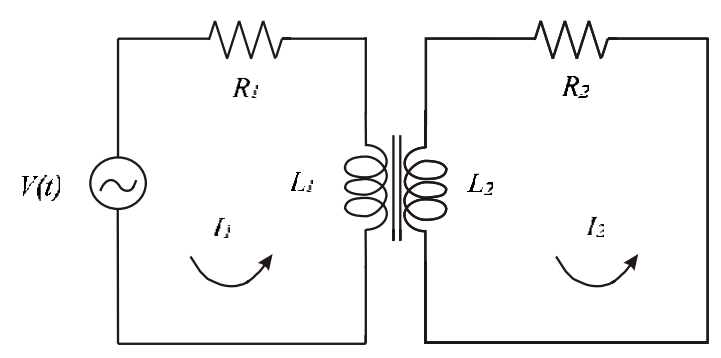

Figure 1: Electric circuit.

We consider the admissible initial condition $x(0)=(0,0)^{T}$, and for this problem we have

$$
\bar{E}=\left(\begin{array}{cc}
-\frac{L_{1}}{R_{1}} & -\frac{\sqrt{L_{1} L_{2}}}{R_{1}} \\
-\frac{\sqrt{L_{1} L_{2}}}{R_{2}} & -\frac{L_{2}}{R_{2}}
\end{array}\right), \quad \bar{f}(t)=\left(\begin{array}{c}
-\frac{V}{R_{1}} \sin (w t) \\
0
\end{array}\right)
$$


Particularizing the numerical scheme (14) for this case we have

$$
x_{n}=\left(I+\Delta t \bar{E}^{D}\right) \bar{E}^{D} \bar{E} x_{n-1}+\left(I+\Delta t \bar{E}^{D}\right) \bar{E}^{D} \bar{f}_{n-1} \Delta t-\left(I-\bar{E}^{D} \bar{E}\right) \bar{f}_{n} .
$$

We will call $S 1$ to this numerical scheme.

On the other hand, particularizing the numerical scheme (15) we have

$$
x_{n}=\left(I+\Delta t \bar{E}^{D}\right) x_{n-1}+\Delta t \bar{E}^{D} \bar{f}_{n-1}-\left(I-\bar{E}^{D} \bar{E}\right)\left(\bar{f}_{n}-\bar{f}_{n-1}\right) .
$$

Similarly, we will call $S 2$ to this numerical scheme.

The analytical solution of the initial value problem associated with system (17) can be obtained by analytic shuffle method [1], and it is given by

$$
\begin{aligned}
x_{1}(t)= & \frac{V}{D}\left(L_{1} R_{2}^{2} w e^{-\frac{R_{1} R_{2} t}{L_{2} R_{1}+L_{1} R_{2}}}-L_{1} R_{2}^{2} w \cos (w t)+\right. \\
& \left.\left(R_{1} R_{2}^{2}+L_{2}^{2} R 1 w^{2}+L_{1} L_{2} R_{2} w^{2}\right) \sin (w t)\right), \\
x_{2}(t)= & -\frac{\sqrt{L_{1} L_{2}} v w}{D}\left(-R_{1} R_{2} e^{-\frac{R_{1} R_{2} t}{L_{2} R_{1}+L_{1} R_{2}}}+R_{1} R_{2} \cos (w t)\right. \\
& \left.+\left(L_{2} R_{1}+L_{1} R_{2}\right) w \sin (w t)\right),
\end{aligned}
$$

where $D=2 L_{1} L_{2} R_{1} R_{2} w^{2}+L_{1}^{2} R_{2}^{2} w^{2}+R_{1}^{2}\left(R_{2}^{2}+L_{2}^{2} w^{2}\right)$.

We have considered the numerical values for the elements of the circuit presented in Table 1.

\begin{tabular}{|c|c|c|c|c|c|}
\hline$L_{1}$ & $L_{2}$ & $R_{1}$ & $R_{2}$ & $V$ & $w$ \\
\hline $20 \mathrm{H}$ & $1 \mathrm{H}$ & $100 \Omega$ & $200 \Omega$ & $220 \mathrm{~V}$ & $100 \pi$ \\
\hline
\end{tabular}

Table 1: Numerical values for the elements of the circuit.

In this example it is easy to see that the continuous system is BIBO stable and the stability property for the numerical methods depends on $\Delta t$. 
We now study the spectrum of the matrices of the two proposed numerical schemes. For the scheme $S 1$ the eigenvalues of matrix $\Delta t \bar{E}^{D}+\bar{E}^{D} \bar{E}$ are $\{1-4.878 \Delta t, 0\}$ then the system is BIBO if we take $0<\Delta t<0.41$. For the scheme $S 2$, the eigenvalues of matrix $\Delta t \bar{E}^{D}+I$ are $\{1-4.878 \Delta t, 1\}$. Thus, we obtain that both methods are BIBO if we take $0<\Delta t<0.41$.

In order to study the performance of the numerical schemes $S 1$ and $S 2$ we have computed the numerical solution of the system (17) with initial condition $x(0)=(0,0)^{T}$ using $\Delta t=0.002,0.001$ and 0.0005 comparing the obtained results with the analytical solutions (20). These results are shown in Figures 2, 3 and 4.
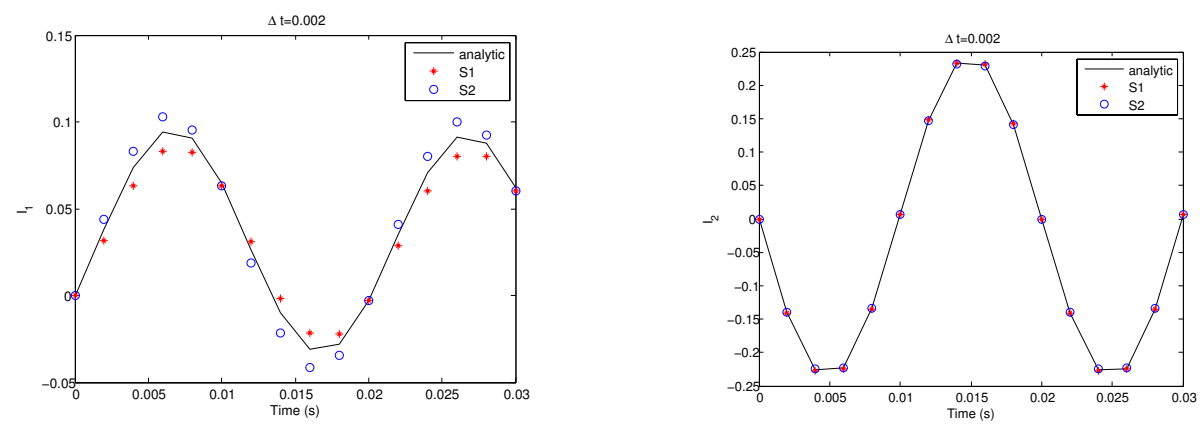

Figure 2: Numerical computation of $I_{1}$ and $I_{2}$ using $\Delta t=0.002$.

We observe that both schemes $S 1$ and $S 2$ provide very good results for the evolution of $I_{2}$ and the numerical results obtained for $I_{1}$ converge to the analytical results when $\Delta t$ approaches to zero.

\section{$5.2 \quad$ Second example}

It is important to remark that the numerical methods presented above are general for any index of the singular system. They depend on the index of matrix $\bar{E}$. In the first example we have presented, matrix $E$ has index 1 and 

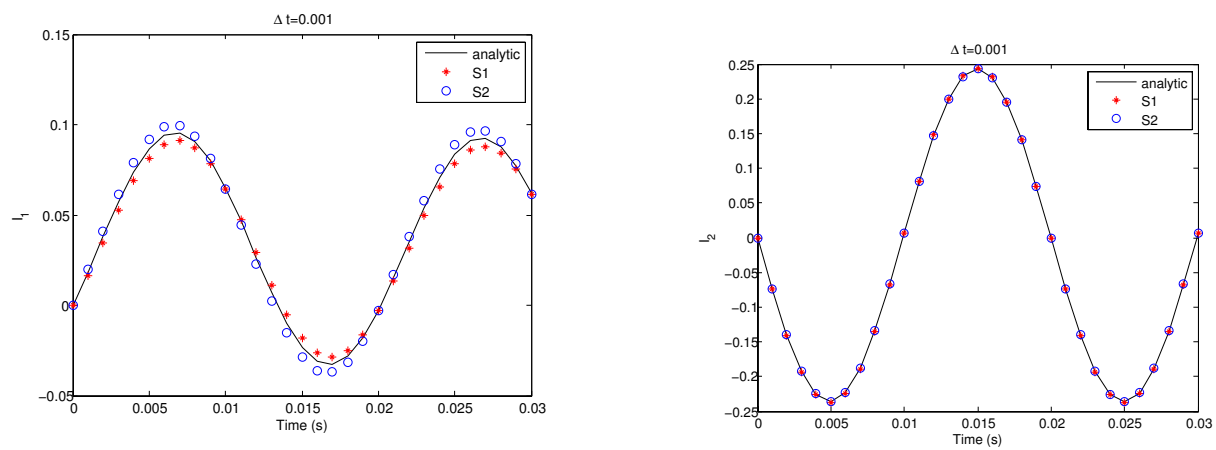

Figure 3: Numerical computation of $I_{1}$ and $I_{2}$ using $\Delta t=0.001$.
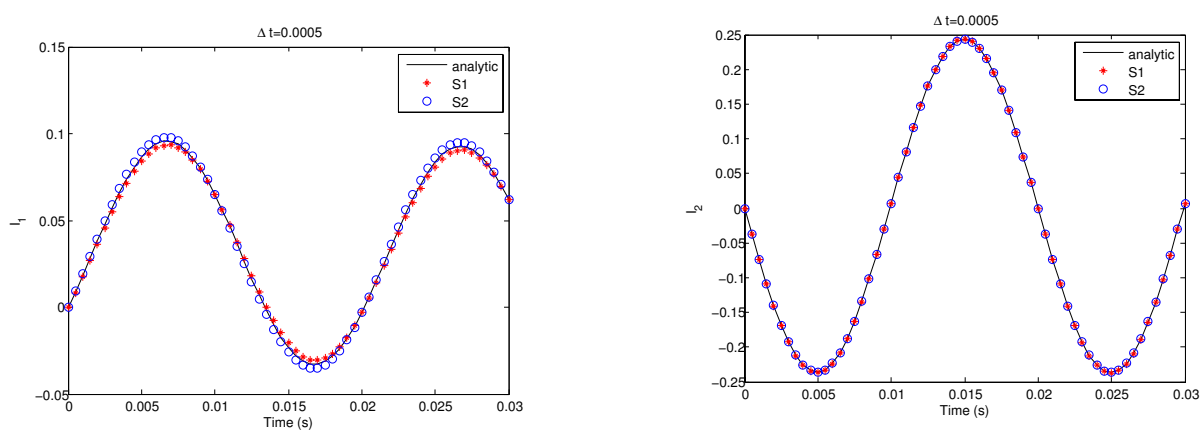

Figure 4: Numerical computation of $I_{1}$ and $I_{2}$ using $\Delta t=0.0005$ for the first example.

the matrix $\bar{E}$ has also index 1, but, in general, it does not exist a relation between those indices.

We consider a second example of a singular system of index 2. This 
system is given by

$$
\left(\begin{array}{llll}
1 & 0 & 0 & 0 \\
0 & 0 & 1 & 0 \\
0 & 0 & 0 & 0 \\
0 & 0 & 0 & 0
\end{array}\right)\left(\begin{array}{c}
x_{1}^{\prime} \\
x_{2}^{\prime} \\
x_{3}^{\prime} \\
x_{4}^{\prime}
\end{array}\right)=\left(\begin{array}{cccc}
0 & 1 & 0 & 0 \\
1 & 0 & 0 & 0 \\
-1 & 0 & 0 & 1 \\
0 & 1 & 1 & 1
\end{array}\right)\left(\begin{array}{c}
x_{1} \\
x_{2} \\
x_{3} \\
x_{4}
\end{array}\right)+\left(\begin{array}{c}
0 \\
0 \\
0 \\
\sin (t)
\end{array}\right) .
$$

We consider the admissible initial condition $x(0)=(0,0,0,0)^{T}$, and for this problem we have

$$
\bar{E}=\left(\begin{array}{rrrr}
0 & 0 & 1 & 0 \\
1 & 0 & 0 & 0 \\
-1 & 0 & -1 & 0 \\
0 & 0 & 1 & 0
\end{array}\right), \quad \bar{f}(t)=\left(\begin{array}{c}
0 \\
0 \\
\sin (t) \\
0
\end{array}\right)
$$

Matrix $\bar{E}$ satisfies $\operatorname{rank}\left(\bar{E}^{2}\right)=\operatorname{rank}(\bar{E})$, then the index of $\bar{E}$ is 1 . Since, $\bar{E}$ has index 1, the numerical schemes (18) and (19) are also valid for this second example.

The analytical solution of the initial value problem associated with system (21) is given by

$$
\left(\begin{array}{l}
x_{1}(t) \\
x_{2}(t) \\
x_{3}(t) \\
x_{4}(t)
\end{array}\right)=\left(\begin{array}{c}
-\sin (t)+\frac{2}{\sqrt{3}} e^{-\frac{t}{2}} \sin \left(\frac{\sqrt{3}}{2} t\right) \\
-\cos (t)+\frac{1}{\sqrt{3}} e^{-\frac{t}{2}}\left(\sqrt{3} \cos \left(\frac{\sqrt{3}}{2} t\right)-\sin \left(\frac{\sqrt{3}}{2} t\right)\right) \\
\cos (t)-\frac{1}{\sqrt{3}} e^{-\frac{t}{2}}\left(\sqrt{3} \cos \left(\frac{\sqrt{3}}{2} t\right)+\sin \left(\frac{\sqrt{3}}{2} t\right)\right) \\
-\sin (t)+\frac{2}{\sqrt{3}} e^{-\frac{t}{2}} \sin \left(\frac{\sqrt{3}}{2} t\right)
\end{array}\right) .
$$


In this example it is easy to see that the continuous system is BIBO stable and the stability property for the numerical methods depends on $\Delta t$. Using the spectrum of the matrices of the numerical schemes $S 1$ and $S 2$ we obtain that both methods are BIBO for $0<\Delta t<1$.

In order to study the performance of the numerical schemes $S 1$ and $S 2$ we have computed the numerical solution of the system (21) with initial condition $x(0)=(0,0,0,0)^{T}$ using $\Delta t=0.2$. The obtained results for $x_{1}$ and $x_{2}$ compared with the analytical solutions (22) are shown in Figure 5. The behavior of components $x_{3}$ and $x_{4}$ is very similar to the behaviour of $x_{1}$ and $x_{2}$.
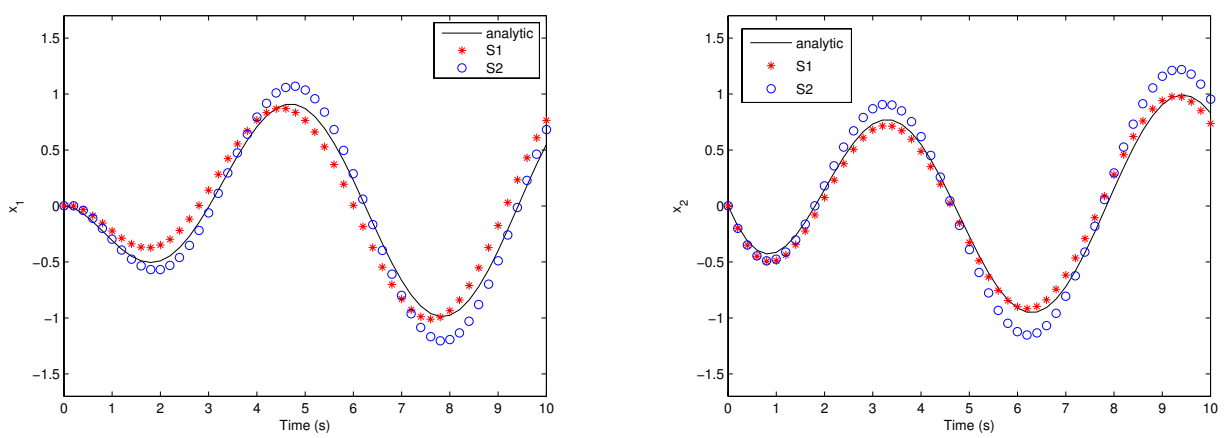

Figure 5: Numerical computation of $x_{1}$ and $x_{2}$ using $\Delta t=0.2$ for the second example.

\subsection{Third example}

As we have already mentioned, in general, there is no relationship between the indices of matrix $E$ and matrix $\bar{E}$, (see, for example, [9]). The third 
example we have studied is given by

$$
\left(\begin{array}{llll}
1 & 0 & 0 & 0 \\
0 & 0 & 1 & 0 \\
0 & 0 & 0 & 0 \\
0 & 0 & 0 & 0
\end{array}\right)\left(\begin{array}{c}
x_{1}^{\prime} \\
x_{2}^{\prime} \\
x_{3}^{\prime} \\
x_{4}^{\prime}
\end{array}\right)=\left(\begin{array}{cccc}
0 & 1 & 0 & 0 \\
1 & 0 & 0 & 0 \\
-1 & 0 & 0 & 1 \\
0 & 0 & 1 & 1
\end{array}\right)\left(\begin{array}{c}
x_{1} \\
x_{2} \\
x_{3} \\
x_{4}
\end{array}\right)+\left(\begin{array}{c}
0 \\
0 \\
0 \\
\sin (t)
\end{array}\right) .
$$

In this new example, index of matrix $E$ is 2 , but matrix $\bar{E}$ satisfies $\operatorname{rank}\left(\bar{E}^{3}\right)=\operatorname{rank}\left(\bar{E}^{2}\right)$, then the index of $\bar{E}=2$. Since the index of $\bar{E}$ is equal to 2 , the numerical scheme $S 1$ is written as

$$
\begin{aligned}
x_{n}= & \left(I+\Delta t \bar{E}^{D}\right) \bar{E}^{D} \bar{E} x_{n-1}+\left(I+\Delta t \bar{E}^{D}\right) \bar{E}^{D} \bar{f}_{n-1} \Delta t \\
& -\left(I-\bar{E}^{D} \bar{E}\right)\left(\bar{f}_{n}+\bar{E}\left(\frac{\bar{f}_{n+1}-\bar{f}_{n}}{\Delta t}\right)\right) .
\end{aligned}
$$

The numerical scheme $S 2$ is now

$$
x_{n}=\left(I+\Delta t \bar{E}^{D}\right) x_{n-1}+\Delta t \bar{E}^{D} \bar{f}_{n-1}-\left(I-\bar{E}^{D} \bar{E}\right) G_{n}
$$

where $G_{n}=\bar{f}_{n}-\bar{f}_{n-1}+\bar{E}\left(\frac{\bar{f}_{n+1}-2 \bar{f}_{n}+\bar{f}_{n-1}}{\Delta t}\right)$.

We consider the admissible initial conditions $x(0)=(0,-1,0,0)^{T}$ and for this problem we have

$$
\bar{E}=\left(\begin{array}{rrrr}
0 & 0 & 1 & 0 \\
1 & 0 & 0 & 0 \\
0 & 0 & -1 & 0 \\
0 & 0 & 1 & 0
\end{array}\right), \quad \bar{f}(t)=\left(\begin{array}{c}
0 \\
0 \\
\sin (t) \\
0
\end{array}\right)
$$

The analytical solution of the initial value problem associated with system (23) is given by 


$$
\left(\begin{array}{l}
x_{1}(t) \\
x_{2}(t) \\
x_{3}(t) \\
x_{4}(t)
\end{array}\right)=\left(\begin{array}{c}
\frac{1}{2} e^{-t}-\frac{1}{2} \cos (t)-\frac{1}{2} \sin (t) \\
-\frac{1}{2} e^{-t}-\frac{1}{2} \cos (t)+\frac{1}{2} \sin (t) \\
-\frac{1}{2} e^{-t}+\frac{1}{2} \cos (t)-\frac{1}{2} \sin (t) \\
\frac{1}{2} e^{-t}-\frac{1}{2} \cos (t)-\frac{1}{2} \sin (t)
\end{array}\right) .
$$

In this third example the continuous system is BIBO stable. Using the spectrum of the matrices of the two proposed numerical schemes $S 1$ and $S 2$, we obtain that both methods are BIBO stable for $0<\Delta t<2$.

In order to study the performance of the numerical schemes (24) and (25) we have computed the numerical solution fo $x_{1}$ and $x_{2}$ of the system (23) with initial condition $x(0)=(0,-1,0,0)^{T}$ using $\Delta t=0.2$. The obtained results compared with the analytical solutions (26) are presented in Figure 6 , for components $x_{1}$ and $x_{2}$. The behaviour of components $x_{3}$ and $x_{4}$ is very similar.
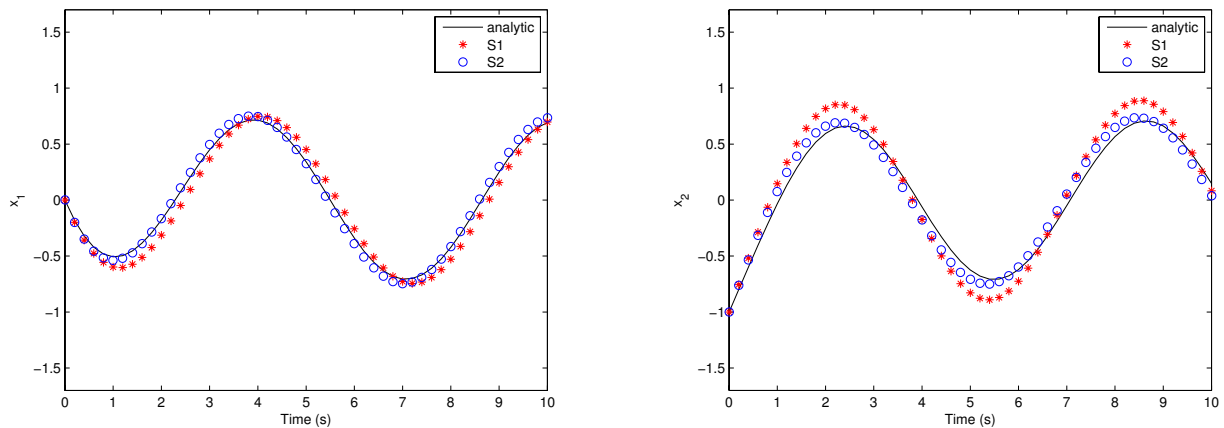

Figure 6: Numerical computation of $x_{1}$ and $x_{2}$ using $\Delta t=0.2$ for the third example. 


\section{Conclusions}

The usual approach to solve a singular system of differential equations consists of converting the system into an ordinary system of differential equations using the shuffle algorithm.

Another possibility is to use implicit backward difference methods. In this paper we study the possibility of developing numerical method for singular systems based on the expression of the analytical solution of a singular linear system in terms of the Drazin inverse of a matrix. The numerical methods proposed are based on the Drazin inverse vector product as the main operation. This operation can be implemented numerically by iterative methods based on the operation matrix vector product, which does not affect the sparsity pattern of the matrices. This fact is very important when DAEs of large dimension are considered. To perform the shuffle algorithm, a triangularization process of the matrices of the system is carried out and this triangularization destroys the possible sparsity pattern of the matrices obtaining, in general, dense matrices, that can be difficult to store in the memory of the computer. In this way the numerical methods proposed are advantageous in terms of memory requirements. Two first order methods are proposed and their performances are studied obtaining the numerical solution of different examples of index 1 and index 2. The obtained results show that the proposed methods work well for singular systems of different indices and the same methodology can be applied to obtain higher order numerical methods. 


\section{Acknowledgements}

This paper has been partially supported by Grant Universitat Politcnica de Valncia, PAID-06-09, Ref.: 2659 and by Grant DGI MTM2010-18228.

\section{References}

[1] K.M. Anstreicher, U.G. Rothblum, "Using Gauss-Jordan elimination to computer the index, generalized nullspace, and Drazin inverse". Linear Algebra and its Applications 85, 221-239, 1987.

[2] P. Benner, V.I. Sokolov, "Partial realization of descriptor systems". System and Control Letters 55 (11), 929-938, 2006.

[3] K.E. Brenan, S.L. Campbell, L.R. Petzold, "Numerical solution of initial-value problems in differential-algebraic equations". SIAM, Philadelphia, 1996.

[4] S.L. Campbell, C.D. Meyer, Jr.. Generalized inverses of linear transformations. Dover, New York (1979).

[5] S.L. Campbell, C.D. Meyer, N.J. Rose "Applications of the Drazin inverse to linear systems of differential equations with singular constant coefficients", SIAM J. Applied Math. 31 (3), 411-425, 1976.

[6] J.J. Climent, M. Neumann, A. Sidi, "A semi-iterative method for real spectrum singular linear systems with an arbitrary index". J. Comput. Appl. Math. 87, 21-38, 1997.

[7] L. Dai. "Singular control systems". Springer-Verlag, New York, USA, 1989. 
[8] F.R. Gantmacher, "The Theory of Matrices". American Mathematical Society, Neew York, USA, 2000.

[9] A. Herrero, A. Ramírez, N. Thome. "An algorithm to check the nonnegativity of singular systems". Applied Mathematics and Computation, 189, 355-365, 2007.

[10] F.L. Lewis, "A survey of linear singular systems", Circuits, Syst. Signal Processing, 5(1), 3-36, 1986.

[11] X. Li, Y. Wei, "Iterative methods for the Drazin inverse of a matrix with a complex spectrum". Applied Mathematics and Computation 147, 855$862,2004$.

[12] D.G. Luenberger, "Time-Invariant Descriptor Systems". Automatica, 14, 473-480, 1978.

[13] A. Sidi, "DGMRES: A GMRES-type algorithm for Drazin-inverse solution of singular nonsymmetric linear systems". Linear Algebra and its Applications, 335, 189-204, 2001.

[14] A. Sidi, Y. Kanevsky, "Orthogonal polynomials and semi-iterative methods for the Drazin-inverse solution of singular linear systems". Numerische Mathematik, 93, 563-581, 2003.

[15] A. Sidi, V. Kluzner, "A BI-CG type iterative method for Drazin-inverse solution of singular inconsistent nonsymmetric linear systems of arbitrary index". Electronic J. of Linear Algebra, 6, 72-94, 2000.

[16] R.F. Sicovec, E.L. Yip, M.A. Epton, "Analysis of Descriptor Systems using Numerical Algorithms". IEEE Trans. Automat. Control, AC26(1), 139-147, 1981. 
[17] G. Wang, Y. Wei, S. Qiao, "Generalized inverses: Theory and computations". Science Press, Shanghai, 2003.

[18] Y. Wei, H. Wu, "The representation and approximation for Drazin inverse". J. Comput. Appl. Math. 126, 417-432, 2000.

[19] E.Y. Yip, R.F. Sicovec, "Solvability, controllability and observability of continuos descriptor systems", IEEE Trans. Automat. Control, AC26(3), 702-707, 1981. 